\title{
Irrigation Water Fluxes in an Agricultural Watershed in Central Oklahoma
}

Sumon Datta1, Saleh Taghvaelan', Mukesh Mehata1, and Daniel Moriasi²

1 Dept. Biosystems \& Agricultural Engineering, OSU, Stillwater, OK 2 USDA-ARS Grazinglands Research laboratory, EI Reno, OK

2019 ASABE International Meeting, Boston, MA July 7-10, 2019 


\section{Background}

$\checkmark$ Irrigation accounts for $41 \%$ of water withdrawal in OK (OWRB)

$\checkmark$ In the Fort Cobb Reservoir Experimental Watershed (FCREW), Irrigation is practiced widely.

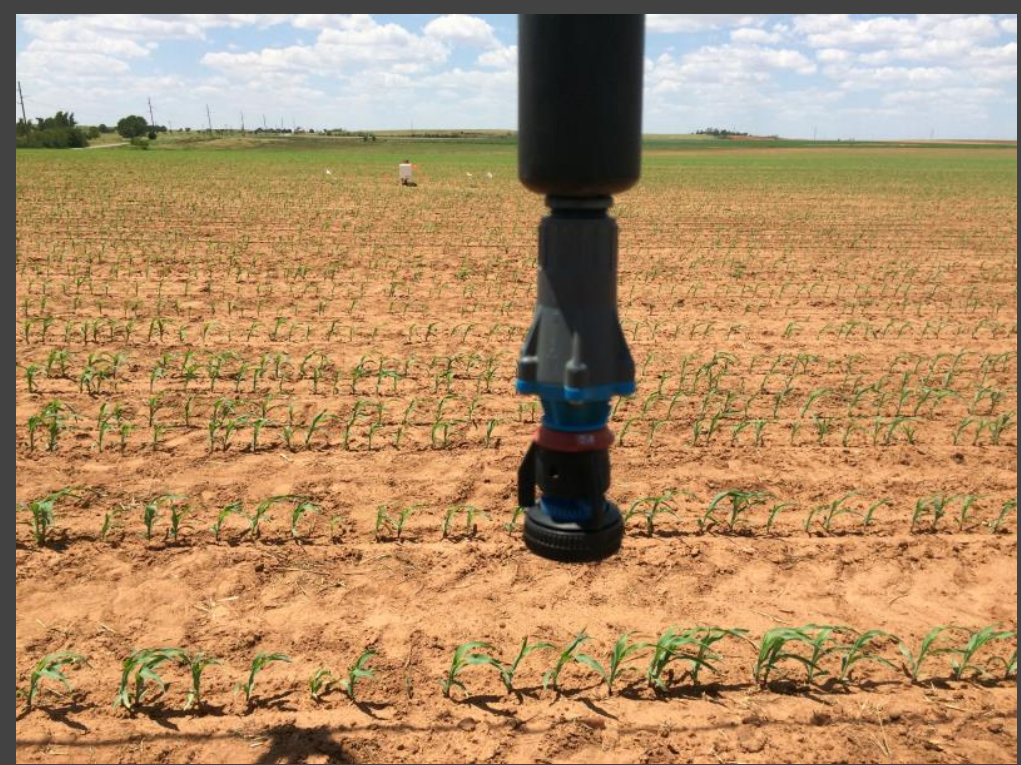

$\checkmark$ The Rush Springs Aquifer is the main source of water (7 ft W.L. decline from 2011 to 2015 during drought).

$\checkmark$ Major crops: peanuts, cotton, soybean, corn, etc. (long history of fertilizer, insecticides application). 


\section{Problem statement}

$\checkmark$ Water availability, especially during droughts

$\checkmark$ Soils are medium- and coarse-textured, highly erodible

$\checkmark$ Applied chemicals

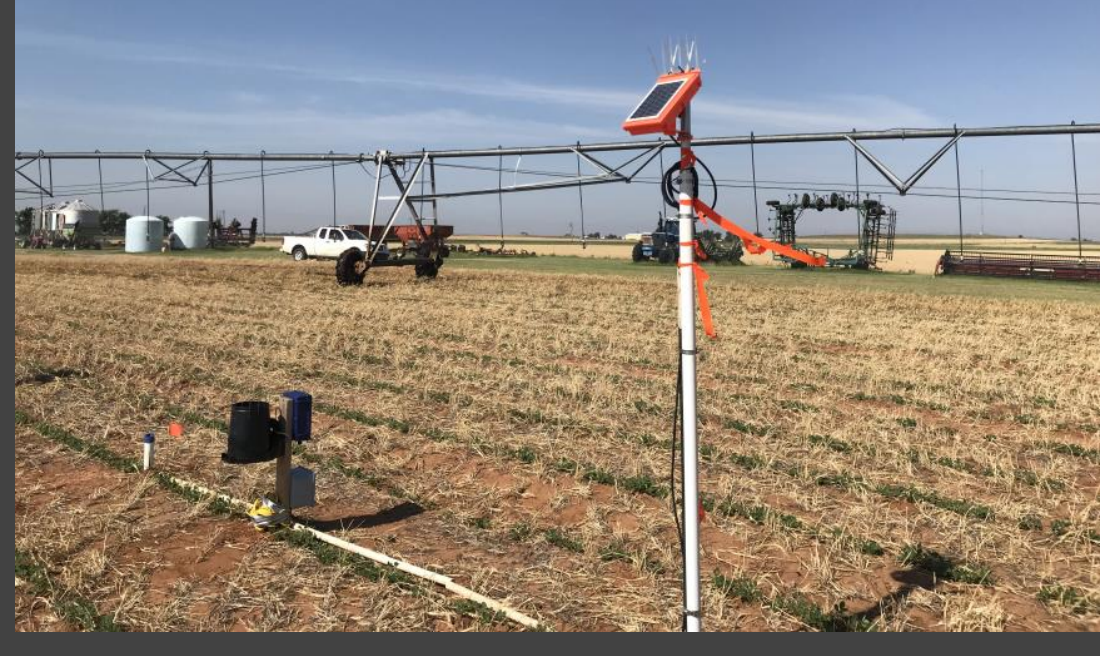
combined with irrigation return flows may be conveyed to downstream land \& water resources.

$\checkmark$ If infiltrated deep enough, groundwater quality may be impaired. 


\section{Solutions}

$\checkmark$ Risk may be assessed by estimating the water fluxes in agricultural fields.

$\checkmark$ Need to estimate or measure important water fluxes: irrigation (I), precipitation $(P)$, runoff $(R O)$, deep percolation (DP), and evapotranspiration (ET)

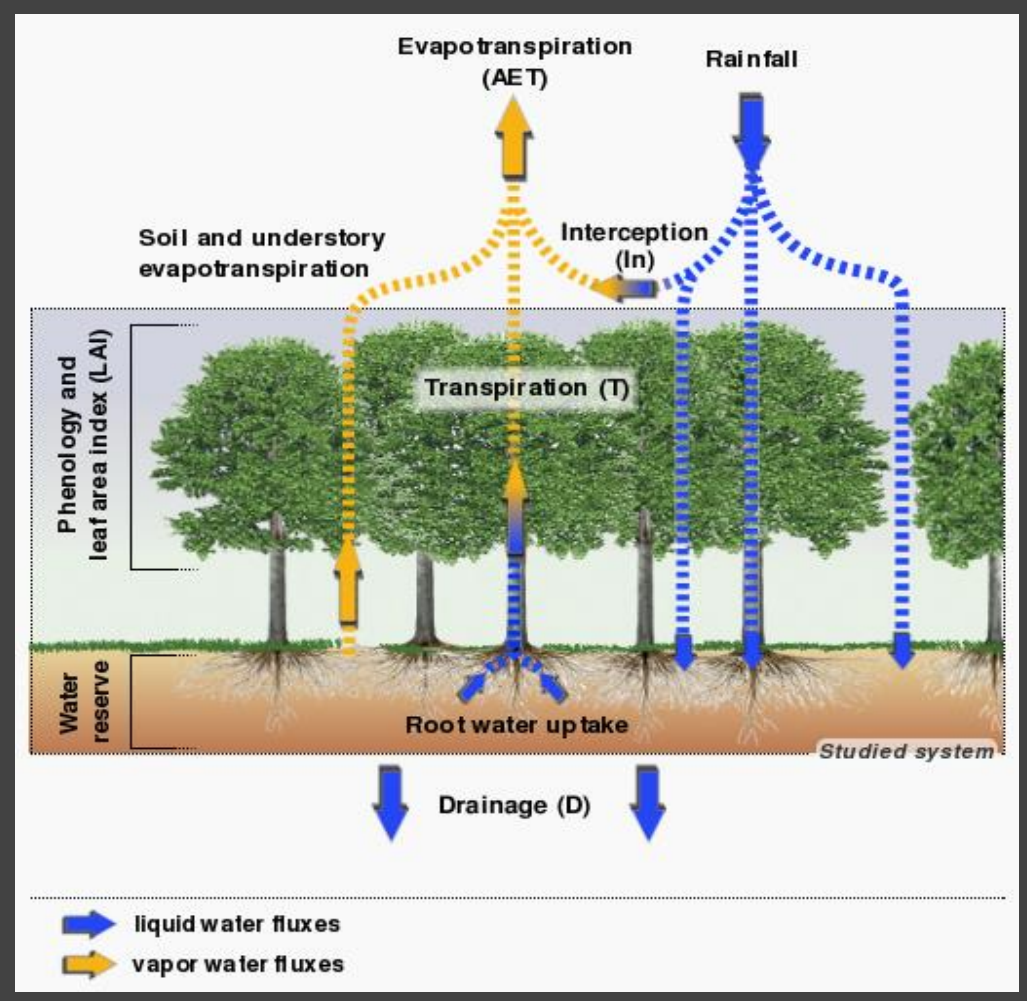




\section{Study Sites}

- FCREW, Central Oklahoma

- 8 commercially irrigated fields in 2017 and 2018

- 3 crops: peanut (PN), Soybean (SB), and Cotton (CT)

- Irrigation system: Center Pivot

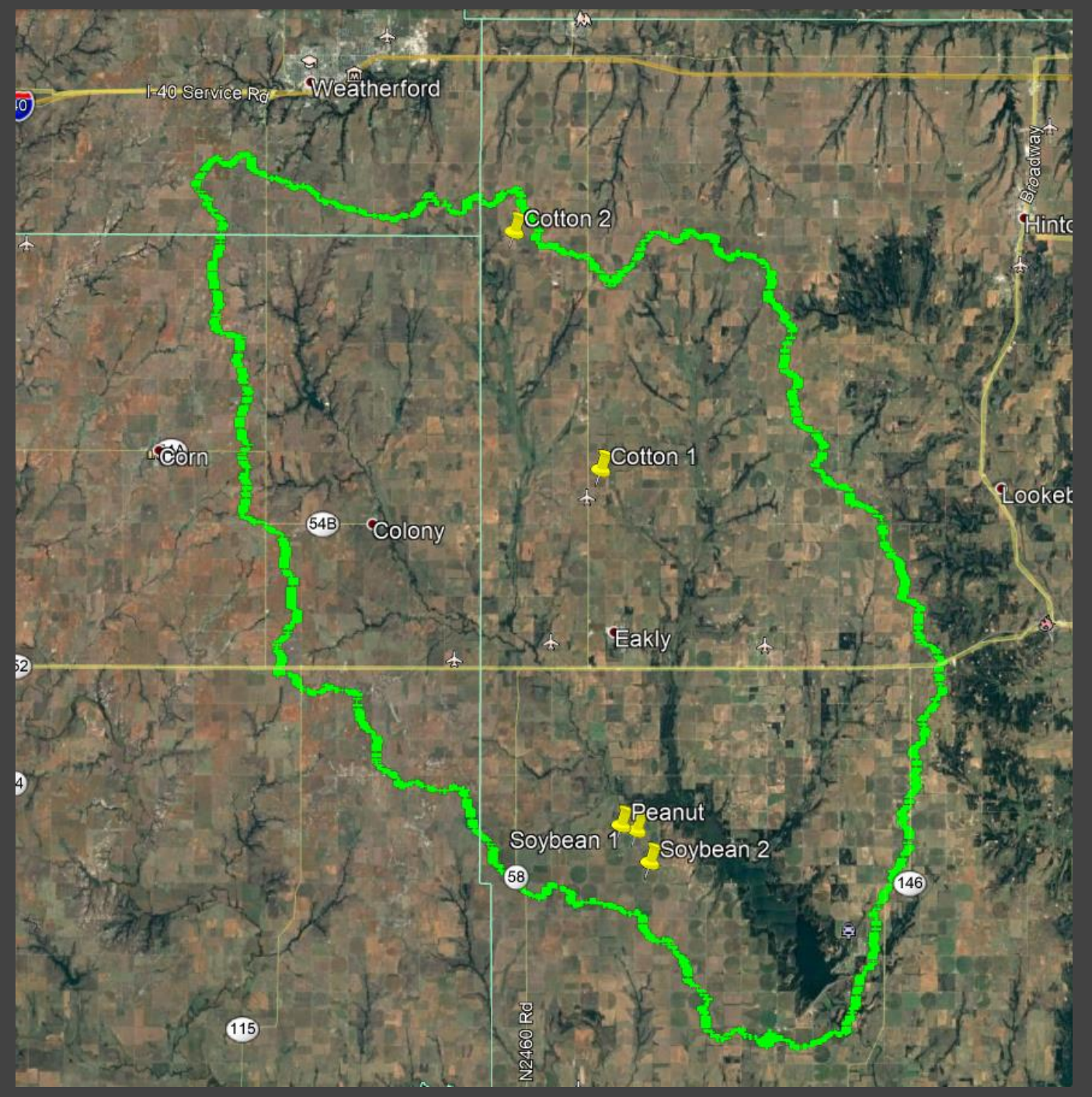




\section{Flux estimation}

$\checkmark$ Fluxes are estimated using simple water balance approach:

$\checkmark \quad \mathrm{DP}=(\mathrm{P}-\mathrm{RO})+\mathrm{I}-\mathrm{ET}_{\mathrm{c} \text { adj }}-\mathrm{D}_{\mathrm{r}}$ (root zone depletion)

$\checkmark$ Combination of measurement and modeling

$\checkmark \quad$ Irrigation and precipitation data from rain gages installed below center-pivot nozzles

$\checkmark$ ET $_{0}$ from Oklahoma Mesonet Weather Data

$\checkmark$ FAO-56 dual crop coefficient method

$\checkmark$ HYDRUS-1D

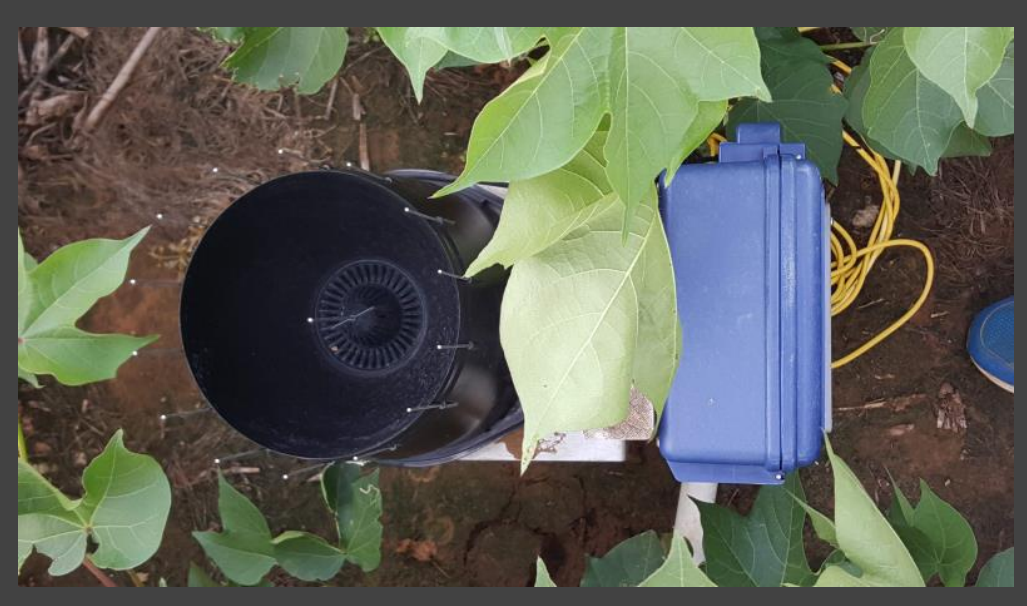




\section{Modeling Framework}

$\checkmark$ Two Oklahoma Mesonet stations

$\checkmark$ ET $_{0}$ : ASCE-PM (Short crop)

$\checkmark$ FAO-56 dual crop coefficient method for estimating adjusted $\mathrm{ET}_{\mathrm{c}}$ (Allen et al. 1998)

$\checkmark \quad \mathrm{ET}_{\mathrm{c} \mathrm{adj}}=\left(\mathrm{K}_{\mathrm{s}} \mathrm{K}_{\mathrm{cb}}+\mathrm{K}_{\mathrm{e}}\right) \mathrm{ET}_{0}$

$\checkmark$ Actual Transpiration $\left(\mathrm{T}_{\mathrm{a}}\right)=\mathrm{K}_{\mathrm{s}} \mathrm{K}_{\mathrm{cb}} \mathrm{ET}_{\mathrm{o}}$

$\checkmark$ Actual Evaporation $\left(E_{\mathrm{a}}\right)=\mathrm{K}_{\mathrm{e}} \mathrm{ET}_{\mathrm{o}}$

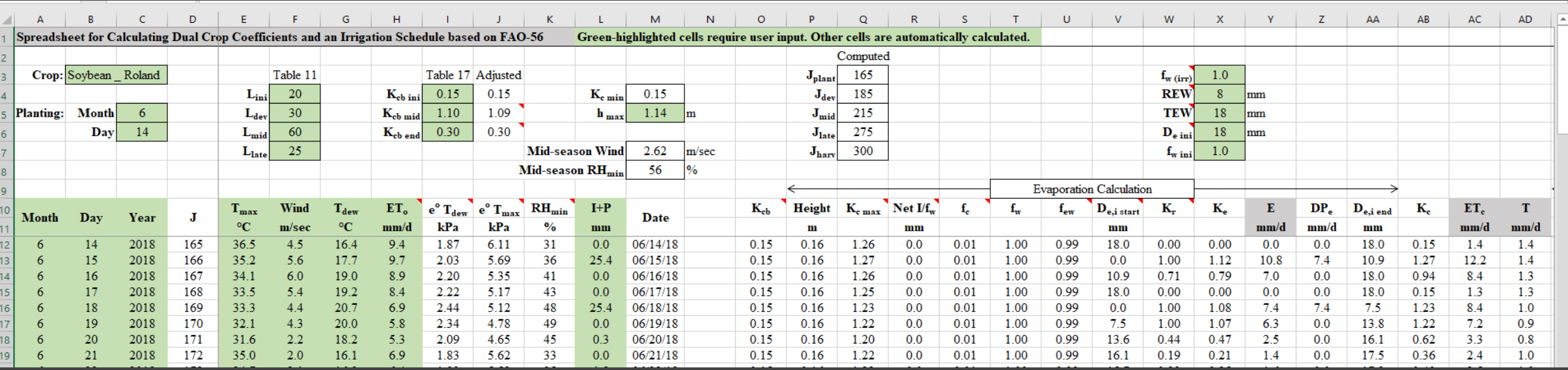

17 Agricultural Sciences 


\section{Modeling Framework}

$\checkmark$ HYDRUS-1D (Simunek et al. 2006):

$\checkmark$ Used $\mathrm{E}_{\mathrm{a}}$ and $\mathrm{T}_{\mathrm{a}}$ from FAO-56

$\checkmark$ Executed model and estimated other fluxes

$\checkmark$ Model Validation

$\checkmark$ Used observed soil moisture data from a locally tested sensors: Acclima TDR315/310S (Datta et al. 2018)

$\checkmark$ Installed at 4 depths $(10,30,51,71 \mathrm{~cm})$ 


\section{Water Fluxes - Cotton (CT) Fields}
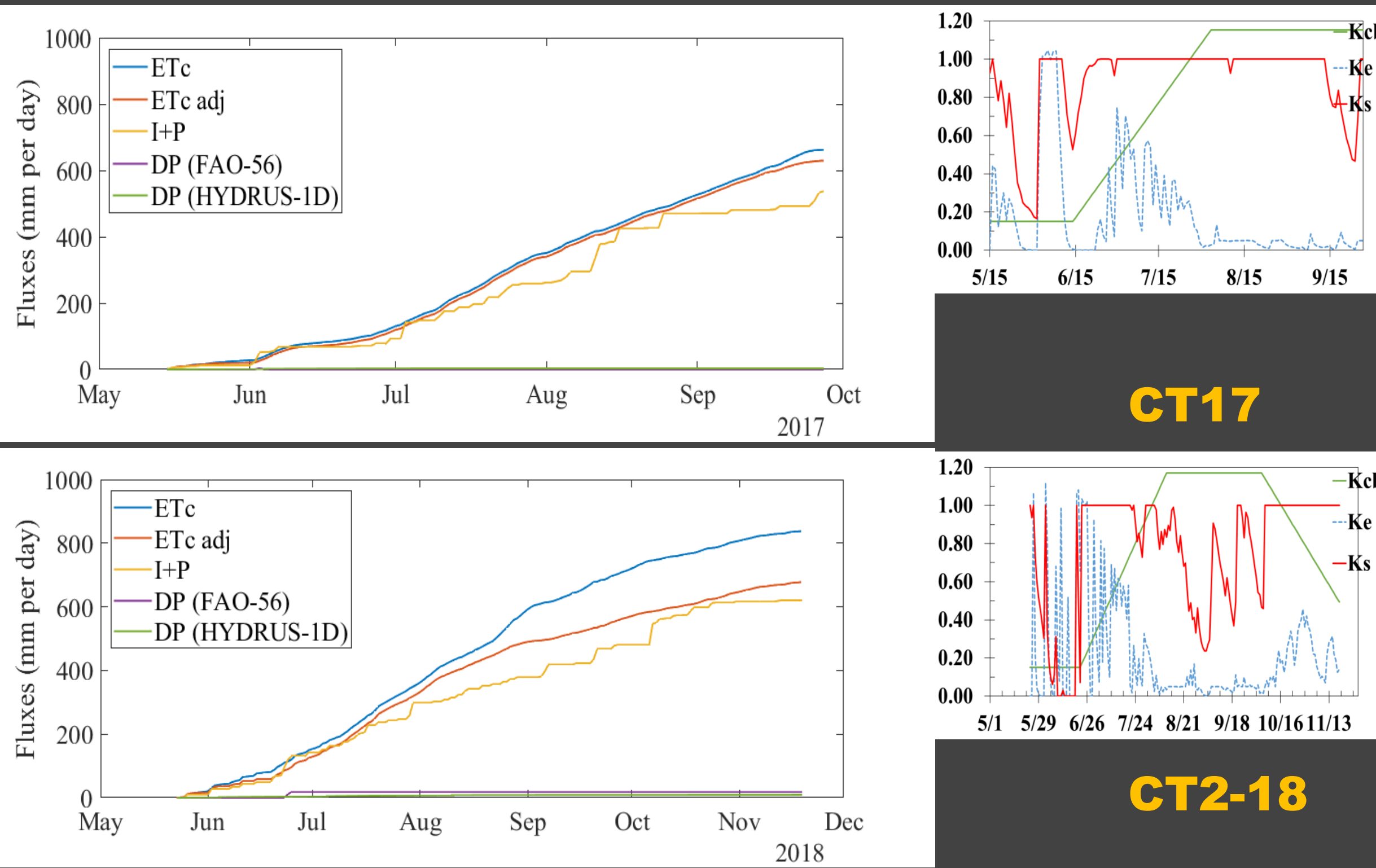

\section{CT2-18}

1
Agricultural Sciences 


\section{Water Fluxes - Soybean (SB) \& Peanut (PN) Fields}
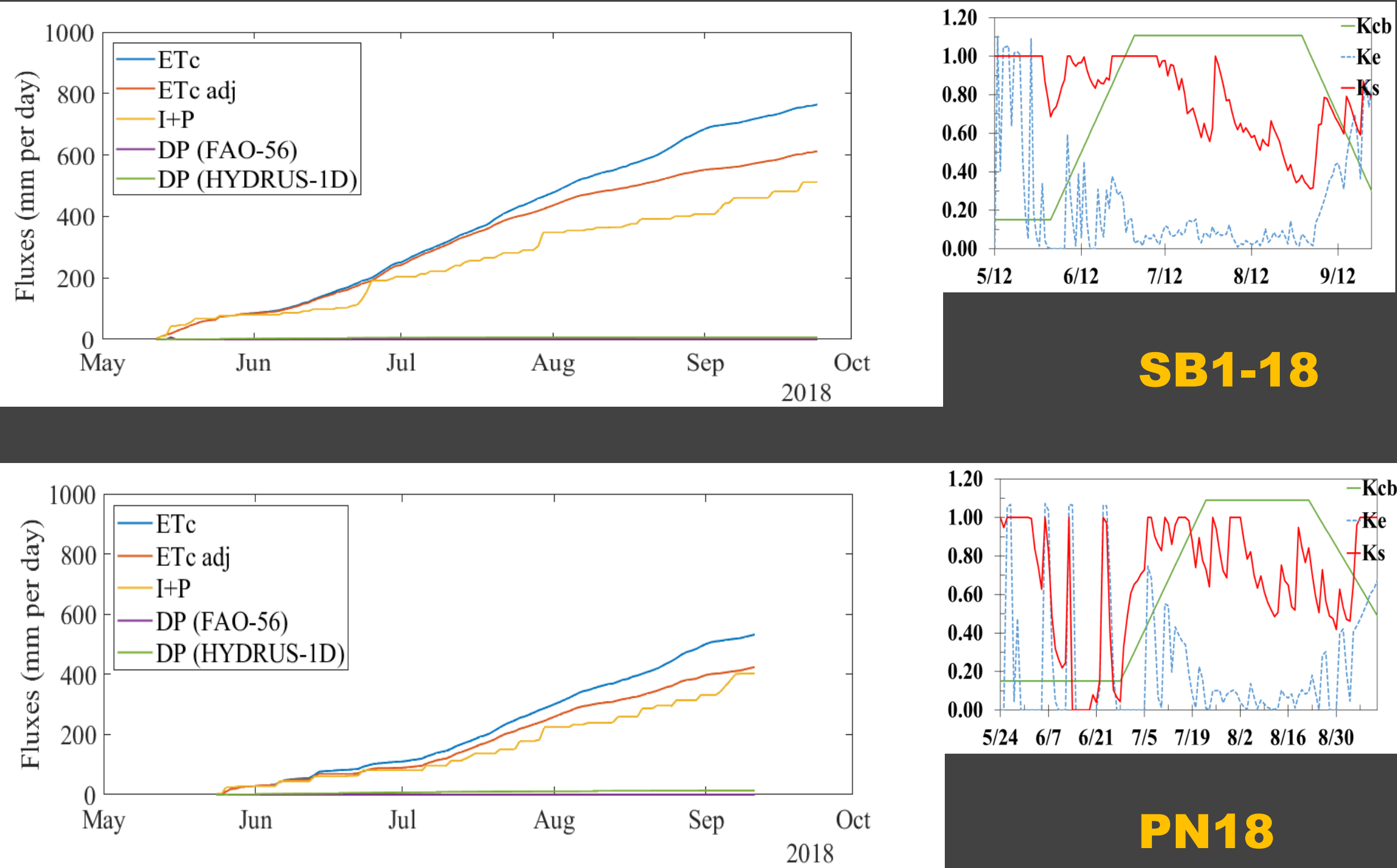

Agricultural Sciences Sives \& Natural Resources Agricultural Engineering 


\section{Validation results}

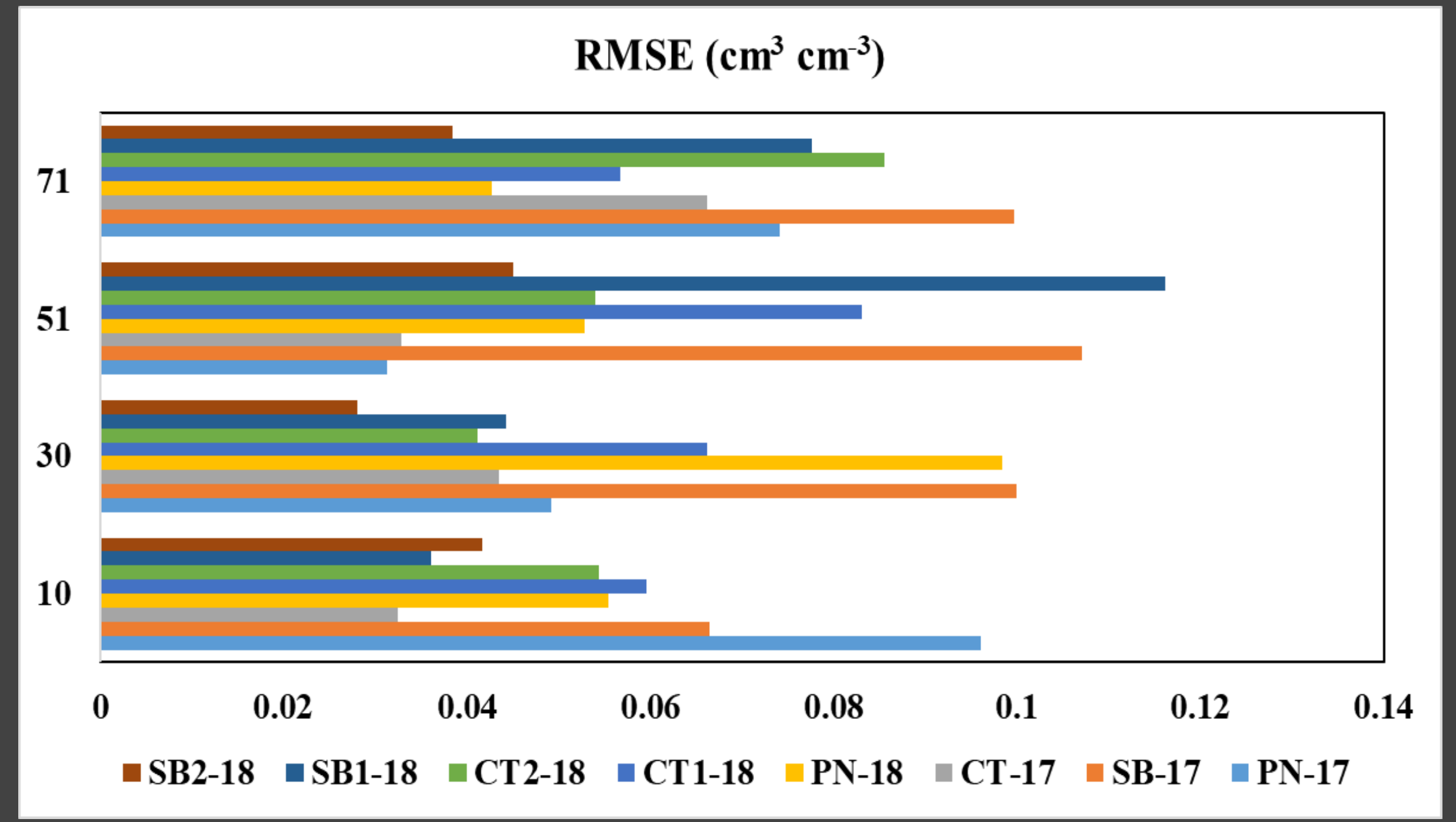

Agricultural Sciences 


\section{Summary of Cumulative Fluxes}

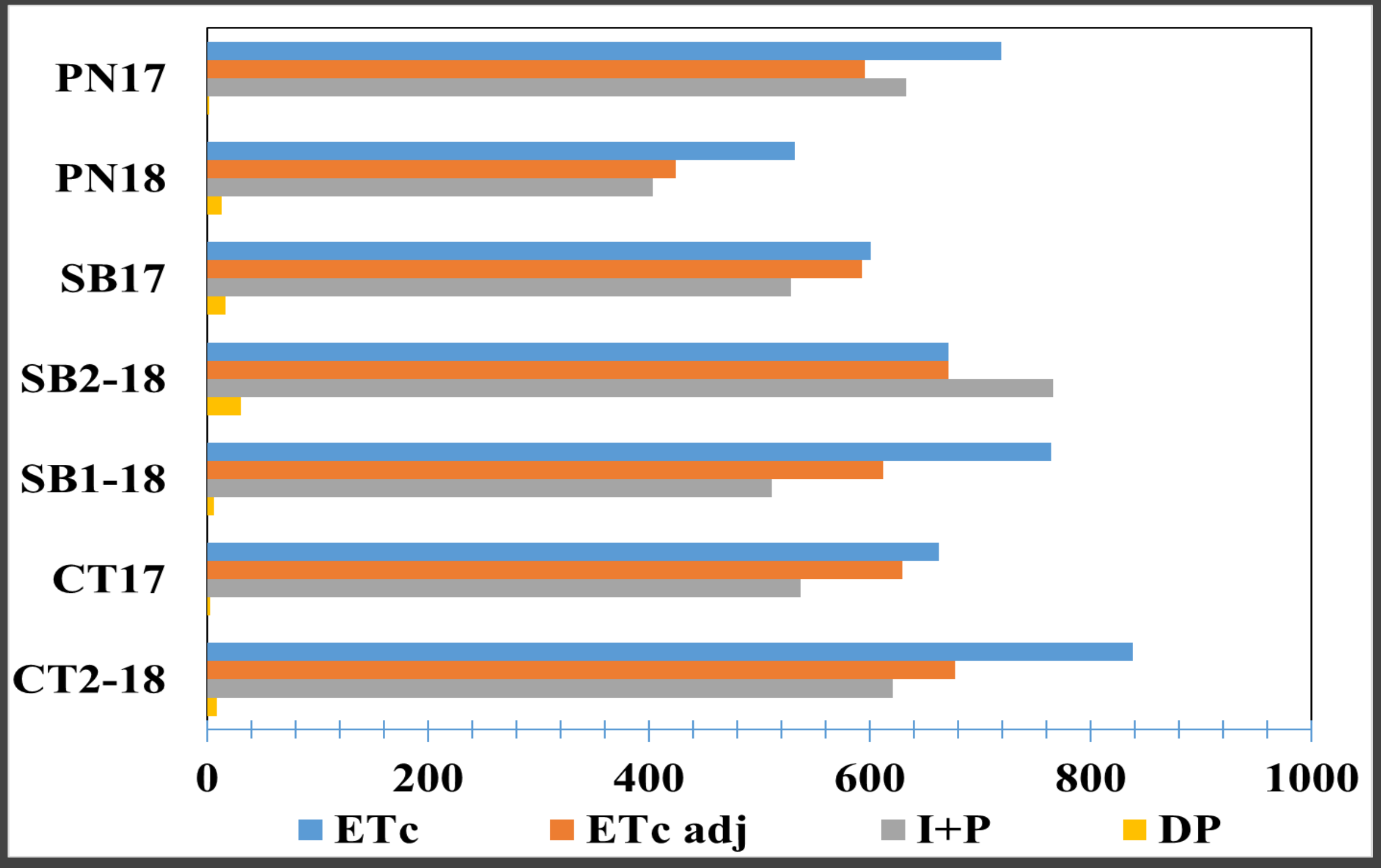

oivision or 


\section{Summary}

- Most fields were under deficit irrigation

- DP was less than $4 \%$ of total water application $(<2 \%$ for most fields)

- Actual irrigations applied were smaller than what should have been applied to maintain stress-free conditions

- Deficit irrigation management by farmers in FCREW

- Continuance of this project to collect more data during different climatic conditions in 2019 


\section{Acknowledgment}

Funding was provided by

- U.S. Geological Survey 104(b) program, administered by the Oklahoma Water Resources Center

- Agricultural Research Service, U.S. Department of Agriculture, Agreement No. 58-3070-5-007
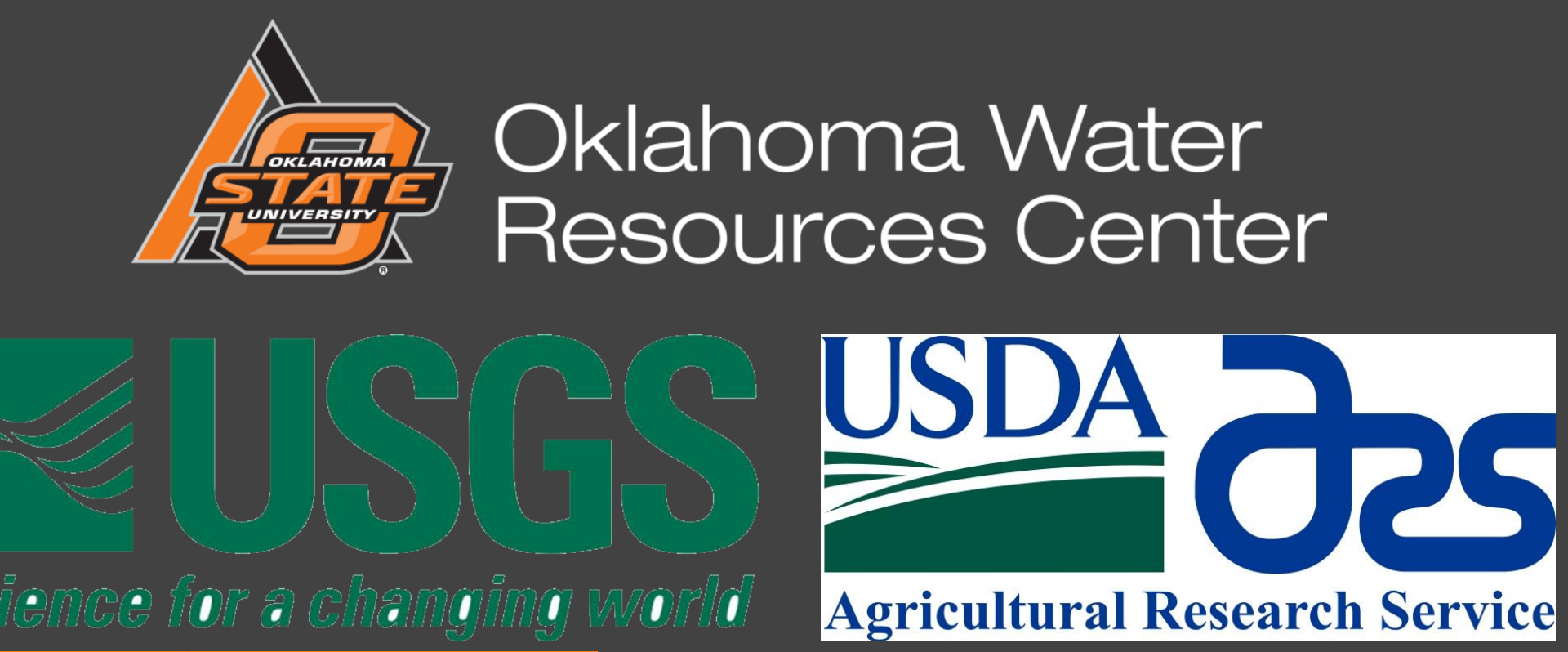


\section{References}

Datta, S., Taghvaeian, S., Ochsner, T., Moriasi, D., Gowda, P., \& Steiner, J. (2018). Performance Assessment of Five Different Soil Moisture Sensors under Irrigated Field Conditions in Oklahoma. Sensors, 18(11), 3786.

Allen, R. G., Pereira, L. S., Raes, D., \& Smith, M. (1998). FAO Irrigation and drainage paper No. 56. Rome: Food and Agriculture Organization of the United Nations, 56(97), e156.

OWRB. Water Facts (Oklahoma Water Resources Board). Available on

Simunek, J., Van Genuchten, M. T., \& Sejna, M. (2006). The HYDRUS software package for simulating the two-and three-dimensional movement of water, heat, and multiple solutes in variably-saturated media. Technical manual, 1. 


\section{DP Estimates}

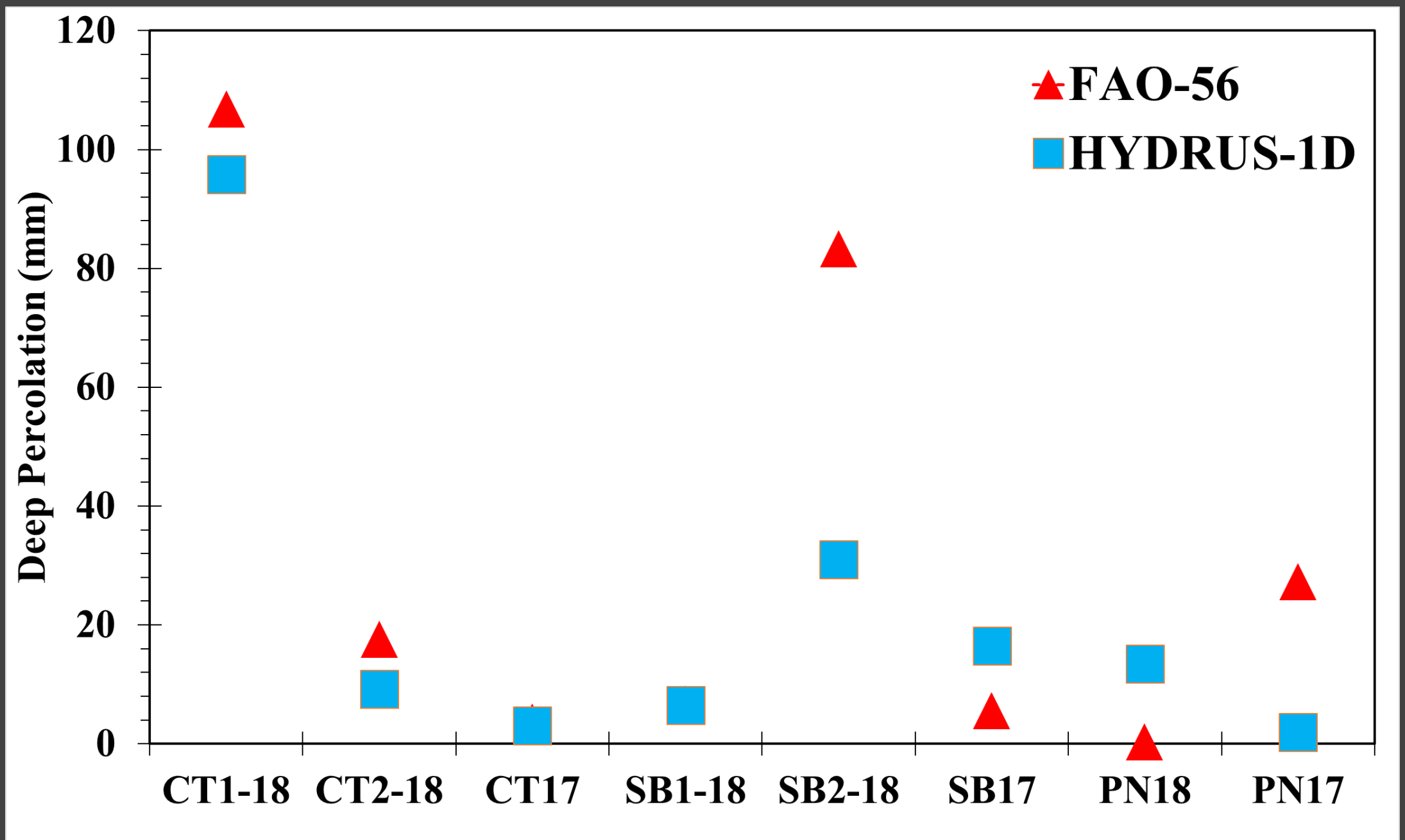

